\title{
Identification of candidate genes in ischemic cardiomyopathy by gene expression omnibus database
}

\author{
Haiming Dang ${ }^{1 \dagger}$, Yicong $\mathrm{Ye}^{2 \dagger}$, Xiliang Zhao ${ }^{2 \dagger}$ and Yong Zeng ${ }^{2^{*}}$ (1)
}

\begin{abstract}
Background: Ischemic cardiomyopathy (ICM) is one of the most usual causes of death worldwide. This study aimed to find the candidate gene for ICM.

Methods: We studied differentially expressed genes (DEGs) in ICM compared to healthy control. According to these DEGs, we carried out the functional annotation, protein-protein interaction (PPI) network and transcriptional regulatory network constructions. The expression of selected candidate genes were confirmed using a published dataset and Quantitative real time polymerase chain reaction (qRT-PCR).

Results: From three Gene Expression Omnibus (GEO) datasets, we acquired 1081 DEGs (578 up-regulated and 503 down-regulated genes) between ICM and healthy control. The functional annotation analysis revealed that cardiac muscle contraction, hypertrophic cardiomyopathy, arrhythmogenic right ventricular cardiomyopathy and dilated cardiomyopathy were significantly enriched pathways in ICM. SNRPB, BLM, RRS1, CDK2, BCL6, BCL2L1, FKBP5, IPO7, TUBB4B and ATP1A1 were considered the hub proteins. PALLD, THBS4, ATP1A1, NFASC, FKBP5, ECM2 and BCL2L1 were top six transcription factors (TFs) with the most downstream genes. The expression of 6 DEGs (MYH6, THBS4, BCL6, BLM, IPO7 and SERPINA3) were consistent with our integration analysis and GSE116250 validation results.
\end{abstract}

Conclusions: The candidate DEGs and TFs may be related to the ICM process. This study provided novel perspective for understanding mechanism and exploiting new therapeutic means for ICM.

Keywords: Ischemic cardiomyopathy, Differentially expressed genes, Gene expression omnibus datasets, Integrated analysis

\section{Background}

Ischemic cardiomyopathy (ICM), a common secondary cardiomyopathy, is one of the most common causes of death worldwide [1]. ICM is a special type or later stage of coronary heart disease, caused by coronary atherosclerotic heart disease, but can also be caused by repeated coronary spasm, coronary inflammation and

\footnotetext{
* Correspondence: anzhenzy@163.com

${ }^{\dagger}$ Haiming Dang, Yicong Ye and Xiliang Zhao contributed equally to this work and should be considered as co-first authors.

${ }^{2}$ Department of cardiology, Capital medical university, Beijing Anzhen hospital, No.2, Anzhen Road, Chaoyan District, Beijing 100029, China Full list of author information is available at the end of the article
}

connective tissue disease. ICM also is the most common cause of heart failure and is associated with significant morbidity and mortality [2]. The main pathophysiological features of ischemic cardiomyopathy are left ventricular enlargement, function of ventricular myocardial diastole and contractile decrease, and further development of congestive heart failure. Like other complex diseases, ICM is caused by interactions of environmental factors and genetic. The factors contributing to ICM are complex, including inflammation, microvessels dysfunction, activation of apoptosis and disruption of $\mathrm{Ca}^{2+}$ homeostasis $[3,4]$. With the emergence of microarray

(c) The Author(s). 2020 Open Access This article is licensed under a Creative Commons Attribution 4.0 International License, which permits use, sharing, adaptation, distribution and reproduction in any medium or format, as long as you give appropriate credit to the original author(s) and the source, provide a link to the Creative Commons licence, and indicate if changes were made. The images or other third party material in this article are included in the article's Creative Commons licence, unless indicated otherwise in a credit line to the material. If material is not included in the article's Creative Commons licence and your intended use is not permitted by statutory regulation or exceeds the permitted use, you will need to obtain permission directly from the copyright holder. To view a copy of this licence, visit http://creativecommons.org/licenses/by/4.0/. The Creative Commons Public Domain Dedication waiver (http://creativecommons.org/publicdomain/zero/1.0/) applies to the data made available in this article, unless otherwise stated in a credit line to the data. 
technology analysis, bioinformatics have become most frequently used means to identify potential biomarkers in a variety of diseases [5-7]. It is reported that a lot of fetal and immediate-early genes are deregulated in the ischemic heart [8]. To our knowledge, many researchers have performed global gene expression to obtain key genes in the underlying mechanisms of ICM [9-11]. For example, Qiao et al. reported that differentially expressed genes (DEGs) and transcription factors (TFs) play pivotal roles in ICM progress through regulating gene expression [12]. Li et al. found that the functional annotation and pathway analysis of DEGs was conducive to further studying the interactions between the differentially expressed genes in ICM [13]. Wang et al. found that PHLDA1 might be a novel molecular marker for ICM [14]. Previous studies identified changes in the protein levels of TFs including GATA4, NFAT1, MEF2C, CSX NKX2-5, NF-kB, STAT-3 and AP-1 in cardiomyopathy and cardiopathy model [15-19]. The molecular mechanism of coordinating transcription in ICM has not been completely understood. Therefore, it is essential to find the pathogenic mechanism and develop new diagnostic biomarker.

The appearance of gene microarray data has become an effective means to search DEGs in multiple diseases which help to reveal underlying mechanisms. Genes that cause complex diseases are always involved in common biological processes in various biological networks [20, 21]. A comprehensive understanding of disease can be

Table 1 Baseline clinical characteristics of subjects

\begin{tabular}{llll}
\hline Characteristics & ICM $(\boldsymbol{n}=10)^{\mathbf{1}}$ & Control $(\mathrm{n}=10)^{\mathbf{1}}$ & $\boldsymbol{P}$ value \\
\hline Gender & & & $>0.90$ \\
$\quad$ Female & $5(50 \%)$ & $5(50 \%)$ & \\
$\quad$ Male & $5(50 \%)$ & $5(50 \%)$ & \\
Age & $60(51,64)$ & $59(58,62)$ & 0.80 \\
TC & $4.12(3.83,5.02)$ & $4.00(3.76,4.33)$ & 0.70 \\
TG & $2(1,3)$ & $1(1,2)$ & 0.50 \\
HDL & $1.16(0.78,1.24)$ & $1.04(0.82,1.26)$ & $>0.90$ \\
LDL & $2.70(2.02,3.14)$ & $2.57(1.77,2.87)$ & 0.70 \\
Hypertension & $3(30 \%)$ & $6(60 \%)$ & 0.40 \\
Diabetes & $5(50 \%)$ & $2(20 \%)$ & 0.30 \\
Hyperlipidemia & $3(30 \%)$ & $6(60 \%)$ & 0.40 \\
Smoking & $7(70 \%)$ & $4(40 \%)$ & 0.40 \\
Drinking & $1(10 \%)$ & $2(20 \%)$ & $>0.90$ \\
HCT & $34(31,41)$ & $39(36,42)$ & 0.13 \\
HB & $110(103,144)$ & $134(122,150)$ & 0.08 \\
\hline
\end{tabular}

1 Statistics presented: $n$ (\%); median (IQR)

2 Statistical tests performed: chi-square test of independence; Wilcoxon ranksum test; Fisher's exact test

Abbreviations: $H D L-C$ high-density lipoprotein cholesterol, $L D L-C$ low-density lipoprotein cholesterol, TG triglyceride, TC Total cholesterol, HCT haematocrit, $H B$ hemoglobin
Table 2 Primer sequences used for real-time PCR

\begin{tabular}{ll}
\hline Primer name & Sequence \\
\hline ACTB-F & CATGTACGTTGCTATCCAGGC \\
ACTB-R & CTCCTTAATGTCACGCACGAT \\
ATP1A1-F & CTGTGGATTGGAGCGATTCTT \\
ATP1A1-R & TTACAACGGCTGATAGCACCA \\
BCL2L1-F & GAGCTGGTGGTTGACTTCTC \\
BCL2L1-R & TCCATCTCCGATTCAGTCCCT \\
FKBP5-F & ACCAAACGGAAAGGAGAGGGA \\
FKBP5-R & TCTTCCCGCTGCATTTCTCC \\
RRS1-F & GGCATCCGTCCCAAGAAGAAG \\
RRS1-R & TTCTTGGCCTGAATCCGCTTG \\
PALLD-F & AAGAAGGCCAGTAGAACTGCT \\
PALLD-R & AAGCGAAGTTTCGTCCAGG \\
ECM2-F & ACAAGCTCTATCACGTCCCG \\
ECM2-R & ACGGTCCATGCCATCATCAG \\
\hline
\end{tabular}

improved by analyzing the biological data of the network module.

Here, the integrated analysis of multiple GEO datasets was performed to identify DEGs between ICM and healthy control. The bioinformatics methods was applied to obtain the ICM related pathways and TFs. The purpose of our study is to better understand the molecular events and pathways of ICM and to develop new therapeutic means for ICM.

\section{Methods}

The analysis of microarray data

The expression profile of ICM and healthy control were downloaded from GEO database (http://www. ncbi.nlm.nih.gov/geo) with the keywords "ischemia" [$\mathrm{MeSH}$ Terms] OR ischemic [All Fields]) AND "cardiomyopathies" [MeSH Terms] OR cardiomyopathy [All Fields]. Three series of datasets, GSE46224 [22], GSE52601 [23] and GSE5406 [24], were selected for data analyses according to the selection criteria described as follows: (1) Dataset should be wholegenome mRNA expression profile by array. (2) Datasets were obtained by left ventricular tissue samples of ICM and healthy control group. (3) The datasets should be normalized or original.

\section{Identification of DEGs}

MetaMA, an R package, is applied to combine data from three GEO datasets. The Benjamini \& Hochberg (False discovery rate; FDR) were used to modulate the $P$-values. The selection criteria for identification of DEGs were: $\mathrm{FDR}<0.05$. The $\mathrm{R}$ package was performed to produce the hierarchical clustering analysis of top 100 DEGs. 
Table 3 Gene expression datasets used in this study

\begin{tabular}{llllll}
\hline GEO accession & Author & Platform & Samples (N:ICM) & Year & Tissue \\
\hline GSE46224 & Kai-Chien Yang [22] & GPL11154lllumina HiSeq 2000 (Homo sapiens) & $8: 8$ & 2014 & Left ventricle apex tissue \\
GSE52601 & Akat KM [23] & GPL10558Illumina HumanHT-12 V4.0 expression beadchip & $4: 3$ & 2014 & left ventricular myocardium \\
GSE5406 & Cappola TP [24] & GPL96 [HG-U133A] Affymetrix Human Genome U133A Array & 16:108 & 2006 Left ventricular myocardium
\end{tabular}

Table 4 The top 40 DEGs in ICM

\begin{tabular}{|c|c|c|c|c|}
\hline ID & Symbol & P.Value & FDR & Regulation \\
\hline 3151 & HMGN2 & 0 & 0 & Up \\
\hline 4060 & LUM & 0 & 0 & Up \\
\hline 54,829 & ASPN & 0 & 0 & Up \\
\hline 57,570 & TRMT5 & 0 & 0 & Up \\
\hline 5654 & HTRA1 & 0 & 0 & Up \\
\hline 25,878 & MXRA5 & 0 & 0 & Up \\
\hline 392 & ARHGAP1 & $1.55 \mathrm{E}-15$ & $1.30 \mathrm{E}-12$ & Up \\
\hline 2487 & FRZB & $3.19 \mathrm{E}-13$ & $2.08 \mathrm{E}-10$ & Up \\
\hline 51,466 & $\mathrm{EVL}$ & $3.92 \mathrm{E}-13$ & $2.30 \mathrm{E}-10$ & Up \\
\hline 4628 & MYH10 & $1.08 \mathrm{E}-12$ & $5.29 \mathrm{E}-10$ & Up \\
\hline 10,947 & AP3M2 & $1.27 \mathrm{E}-12$ & $5.98 \mathrm{E}-10$ & Up \\
\hline 3910 & LAMA4 & $1.45 \mathrm{E}-12$ & $6.30 \mathrm{E}-10$ & Up \\
\hline 3043 & $\mathrm{HBB}$ & $1.56 \mathrm{E}-12$ & $6.54 \mathrm{E}-10$ & Up \\
\hline 1513 & CTSK & 4.13E-12 & 1.56E-09 & Up \\
\hline 1842 & ECM2 & 1.09E-11 & 3.86E-09 & Up \\
\hline 4330 & MN1 & $1.13 \mathrm{E}-11$ & $3.92 \mathrm{E}-09$ & Up \\
\hline 7060 & THBS4 & $1.63 \mathrm{E}-11$ & 5.45E-09 & Up \\
\hline 23,114 & NFASC & $3.06 \mathrm{E}-11$ & $9.22 \mathrm{E}-09$ & Up \\
\hline 57,758 & SCUBE2 & $5.44 \mathrm{E}-11$ & $1.56 \mathrm{E}-08$ & Up \\
\hline 4878 & NPPA & 5.95E-11 & $1.66 \mathrm{E}-08$ & Up \\
\hline 8547 & FCN3 & 0 & 0 & Down \\
\hline 3163 & HMOX2 & 0 & 0 & Down \\
\hline 12 & SERPINA3 & 0 & 0 & Down \\
\hline 28,231 & SLCO4A1 & 0 & 0 & Down \\
\hline 4140 & MARK3 & 0 & 0 & Down \\
\hline 2289 & FKBP5 & 4.44E-16 & 4.01E-13 & Down \\
\hline 84,525 & HOPX & $1.40 \mathrm{E}-13$ & $9.69 \mathrm{E}-11$ & Down \\
\hline 2752 & GLUL & $3.79 \mathrm{E}-13$ & 2.30E-10 & Down \\
\hline 476 & ATP1A1 & 4.67E-13 & $2.45 \mathrm{E}-10$ & Down \\
\hline 262 & AMD1 & $4.50 \mathrm{E}-13$ & $2.45 \mathrm{E}-10$ & Down \\
\hline 10,157 & AASS & $1.42 \mathrm{E}-12$ & $6.30 \mathrm{E}-10$ & Down \\
\hline 27,254 & CSDC2 & $3.51 \mathrm{E}-12$ & $1.42 \mathrm{E}-09$ & Down \\
\hline 4624 & MYH6 & $4.11 \mathrm{E}-12$ & $1.56 \mathrm{E}-09$ & Down \\
\hline 23,022 & PALLD & $6.10 \mathrm{E}-12$ & 2.24E-09 & Down \\
\hline 9057 & SLC7A6 & $2.05 \mathrm{E}-11$ & $6.68 \mathrm{E}-09$ & Down \\
\hline 9588 & PRDX6 & $2.31 \mathrm{E}-11$ & 7.33E-09 & Down \\
\hline 598 & BCL2L1 & $2.91 \mathrm{E}-11$ & $9.00 \mathrm{E}-09$ & Down \\
\hline 5533 & РPP3СC & $3.50 \mathrm{E}-11$ & $1.03 \mathrm{E}-08$ & Down \\
\hline 5604 & MAP2K1 & 7.18E-11 & $1.92 \mathrm{E}-08$ & Down \\
\hline 23,212 & RRS1 & $1.44 \mathrm{E}-10$ & $3.53 \mathrm{E}-08$ & Down \\
\hline
\end{tabular}

\section{Functional annotation}

Gene Ontology (GO) classification and the Kyoto Encyclopedia of Genes and Genomes (KEGG) pathway enrichment analyses were structured by using GeneCodis (http://genecodis.cnb.csic.es/analysis). The terms with $\mathrm{FDR}<0.05$ was significant results.

\section{ICM -specific protein-protein interaction (PPI) network}

The top 50 DEGs in ICM were applied to construct the PPI network by using Biological General Repository for Interaction Datasets (BioGRID) (http://thebiogrid.org/), and then the PPI network was visualized by Cytoscape (3.6.1) (http://www.cytoscape.org/). The nodes represent proteins and edges connect the nodes to show their relationship.

\section{ICM -specific transcriptional regulatory networks}

The corresponding promoters of the top 20 upregulated or down-regulated DEGs were obtained by UCSC (http://genome.ucsc.edu). The TF that regulates these DEGs comes from the matching tool in TRAN SFAC. The ICM -specific transcriptional regulatory network was built by Cytoscape.

\section{Validation in the GEO dataset}

The dataset of GSE116250 [25] was downloaded from the GEO database and used to validate the expression pattern of selected DEGs. The dataset GSE116250 was published on Nov 14, 2018 and examined the left ventricle tissue sample consisting of 13 ICM patients and 14 healthy controls.

\section{Confirmation by qRT-PCR}

Patients presenting to Beijing Anzhen Hospital from July 2018 to December 2018 for coronary angiography were recruited consecutively for the study. Subjects were included in study as cases when left ventricular ejection fraction (LVEF) of $\leq 40 \%$ and fulfilling one of the following criteria: patients with history of myocardial infarction or revascularization (cardiac bypass surgery or percutaneous coronary intervention), patients with $\geq 75 \%$ stenosis of left main or proximal LAD, or patients with $\geq 75 \%$ stenosis of two or more epicardial vessels [26]. Subjects with LVEF of $>50$ and $<50 \%$ stenosis in any main coronary artery were included as controls. 


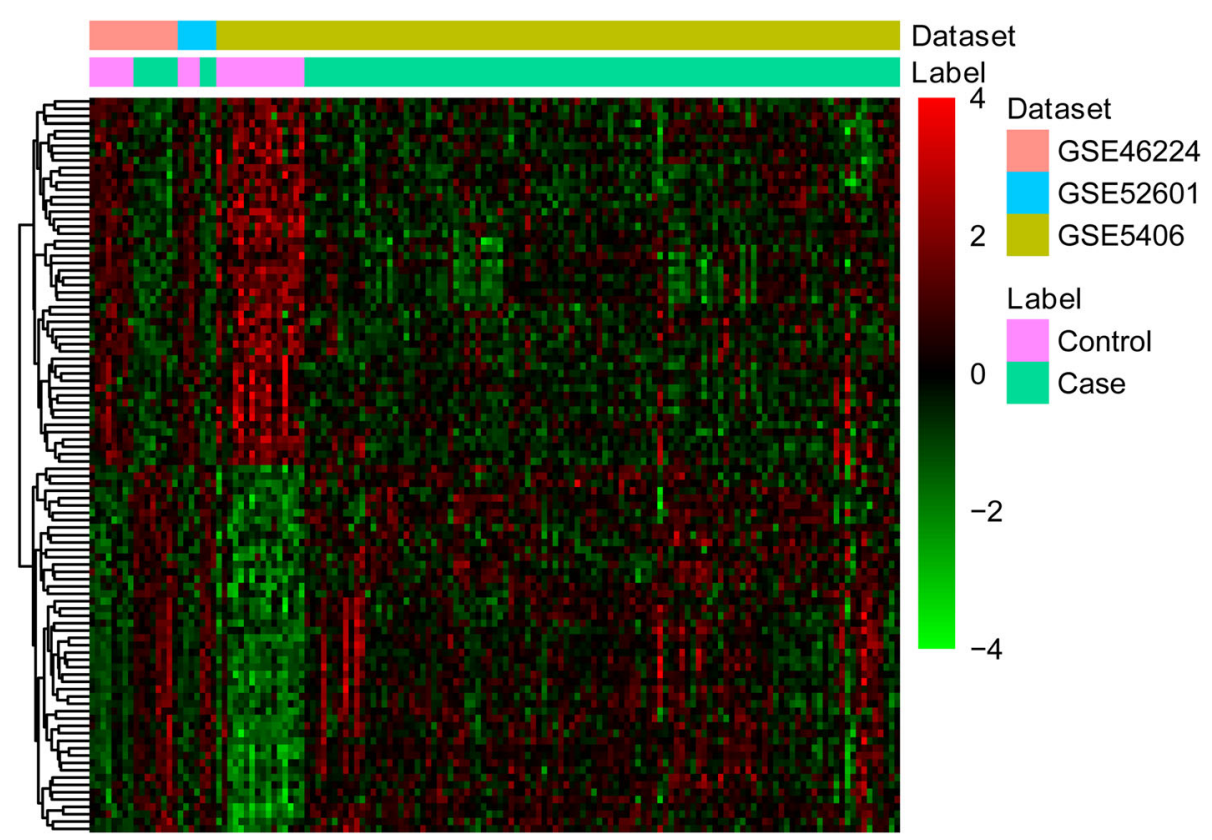

Fig. 1 Heatmap of top 100 DEGs between ICM and healthy controls. Row and column represented DEGs and tissue samples, respectively. Color scale represented normalized expression levels of DEGs between ICM and healthy controls after log2 transformation. Red and green color were used to represent up- and downregulation in ICM, respectively

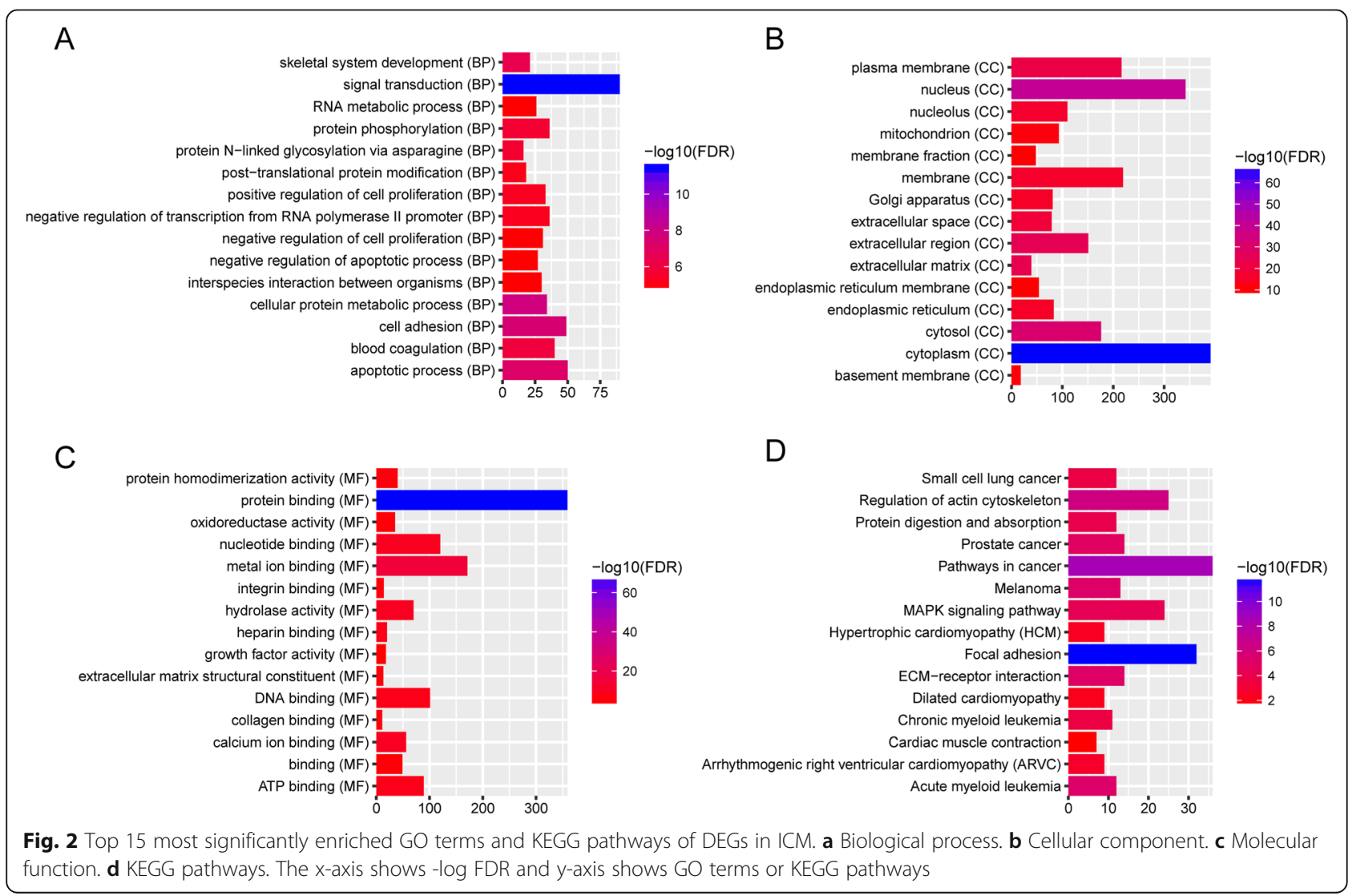


Ten patients diagnosed as ICM and 10 controls were enrolled in this study. The detailed characteristics of the patients were listed in Table 1. All patients were first on an empty stomach for $12 \mathrm{~h}$. Then, we collected the blood samples by venipuncture at 7:00-8:00 of the next morning. This study has been approved by the ethics institute of our hospital. The signed informed consents of all the participants were obtained. Total RNA was isolated with the total RNA kit (Invitrogen, China). Fast Quant RT Kit (Invitrogen, China) was utilized to produce the complementary DNA. Then we performed the GRT-PCR with the Super Real PreMix Plus SYBR Green (Invitrogen, USA) on ABI 7500 real-time PCR system. The amplification process was performed under the following conditions: $15 \mathrm{~min}$ at $95^{\circ} \mathrm{C}$ followed by 40 cycles of $10 \mathrm{~s}$ at $95^{\circ} \mathrm{C}, 30 \mathrm{~s}$ at $55^{\circ} \mathrm{C}, 32 \mathrm{~s}$ at $72^{\circ} \mathrm{C}$, and $15 \mathrm{~s}$ at $95^{\circ} \mathrm{C}, 60 \mathrm{~s}$ at $60^{\circ} \mathrm{C}$, $15 \mathrm{~s}$ extension at $95^{\circ} \mathrm{C}$. The $2-\Delta \Delta \mathrm{Ct}$ method was used to address the data. The PCR primers used are displayed in Table 2 .

\section{Results}

\section{DEGs in ICM}

Three datasets (GSE46224, GSE52601 and GSE5406) were obtained from GEO (Table 3). Compared with the healthy controls, 1081 DEGs (578 genes were upregulated and 503 genes were down-regulated) in ICM were obtained. All DEGs between ICM and healthy controls were displayed in Supplementary Table S1. Top 40 DEGs between ICM and healthy controls were demonstrated in Table 4. Hierarchical clustering of top 100 DEGs was indicated in Fig. 1.

\section{Functional annotation}

Base on the GO enrichment analysis, signal transduction $(\mathrm{FDR}=3.04 \mathrm{E}-12)$, apoptotic process $(\mathrm{FDR}=7.64 \mathrm{E}-08)$, cytoplasm $(\mathrm{FDR}=3.40 \mathrm{E}-67)$ and protein binding $(\mathrm{FDR}=$ 6.06E-70) were most significantly enriched GO terms. After the KEGG pathway enrichment analysis, we found that ECM-receptor interaction (FDR $=8.23 \mathrm{E}-06)$, MAPK signaling pathway $(\mathrm{FDR}=4.96 \mathrm{E}-05)$, Cardiac muscle contraction $(\mathrm{FDR}=0.021418)$, hypertrophic cardiomyopathy
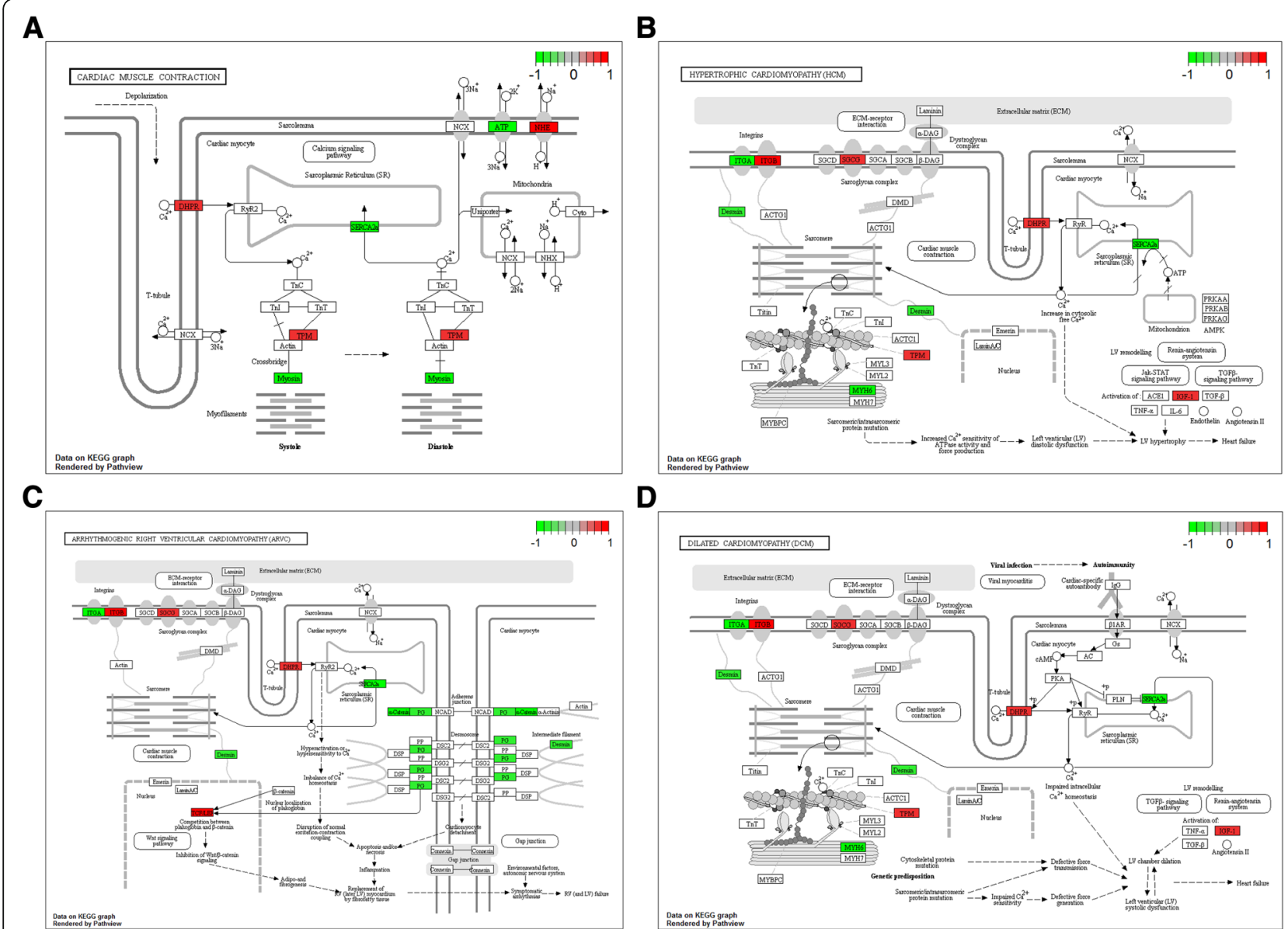

Fig. 3 The key pathway of DEGs enrichment. a The cardiac muscle contraction pathway. b The hypertrophic cardiomyopathy pathway. cThe arrhythmogenic right ventricular cardiomyopathy pathway. $\mathbf{d}$ The dilated cardiomyopathy pathway. The red rectangles were represented the components regulated by the DEGs that enriched in ICM 
$(\mathrm{FDR}=0.003907)$, arrhythmogenic right ventricular cardiomyopathy $(\mathrm{FDR}=0.00204)$ and dilated cardiomyopathy (FDR $=0.005789)$ were significantly enriched pathways in ICM. The top 15 most significantly enriched GO terms and KEGG pathways of DEGs in ICM were listed in Fig. 2 a-d. Pathways of cardiac muscle contraction, hypertrophic cardiomyopathy, arrhythmogenic right ventricular cardiomyopathy and dilated cardiomyopathy were displayed in Fig. 3a, b, c and d, respectively.

\section{ICM -specific PPI network}

The PPI network of top 50 DEGs in ICM was consisted of 208 nodes and 194 edges (Fig. 4). SNRPB (degree = $12)$, BLM $($ degree $=11)$, RRS1 $($ degree $=11)$, CDK2 $($ degree $=9), \quad$ BCL6 $\quad($ degree $=9), \quad$ BCL2L1 $\quad($ degree $=9)$, FKBP5 (degree $=8)$, IPO7 (degree $=8$ ), TUBB4B (degree $=8$ ) and ATP1A1 (degree $=7$ ) were considered the hub proteins.

\section{ICM -specific transcriptional regulatory networks}

According to TRANSFAC, 64 TFs targeting 40 DEGs (top 20 up-regulated or down-regulated genes) were identified.
ICM-specific transcriptional regulatory network was built, which consisted of 104 nodes and 290 edges (Fig. 5). Among of them, PALLD (degree $=17)$, THBS4 (degree $=$ 14), ATP1A1 (degree $=12)$, NFASC (degree $=12)$, FKBP5 $($ degree $=12)$, ECM2 $($ degree $=12)$ and BCL2L1 $($ degree $=$ 10) were top 6 TFs with the most downstream DEGs.

\section{Validation in GSE116250}

Six DEGs (MYH6, THBS4, BCL6, BLM, IPO7 and SERPINA3) were selected to verify in GSE116250 dataset. Among them, BCL6, BLM and IPO7 were the hub gene of ICM-specific PPI network. THBS4 was top TFs covering the most downstream DEGs. MYH6 and SERPINA3 were top 40 DEGs in ICM. As displayed in Fig. 6, the expression of six DEGs were consistent with our integration results. MYH6, BCL6, BLM, IPO7 and SERPINA3 were down-regulated while THBS4 was up-regulated in ICM compared with healthy control.

\section{Validation by $\mathrm{qRT}-\mathrm{PCR}$}

Six DEGs validated in GSE116250 were chose for qRTPCR verification (Fig. 6). As shown in Fig. 7, MYH6,

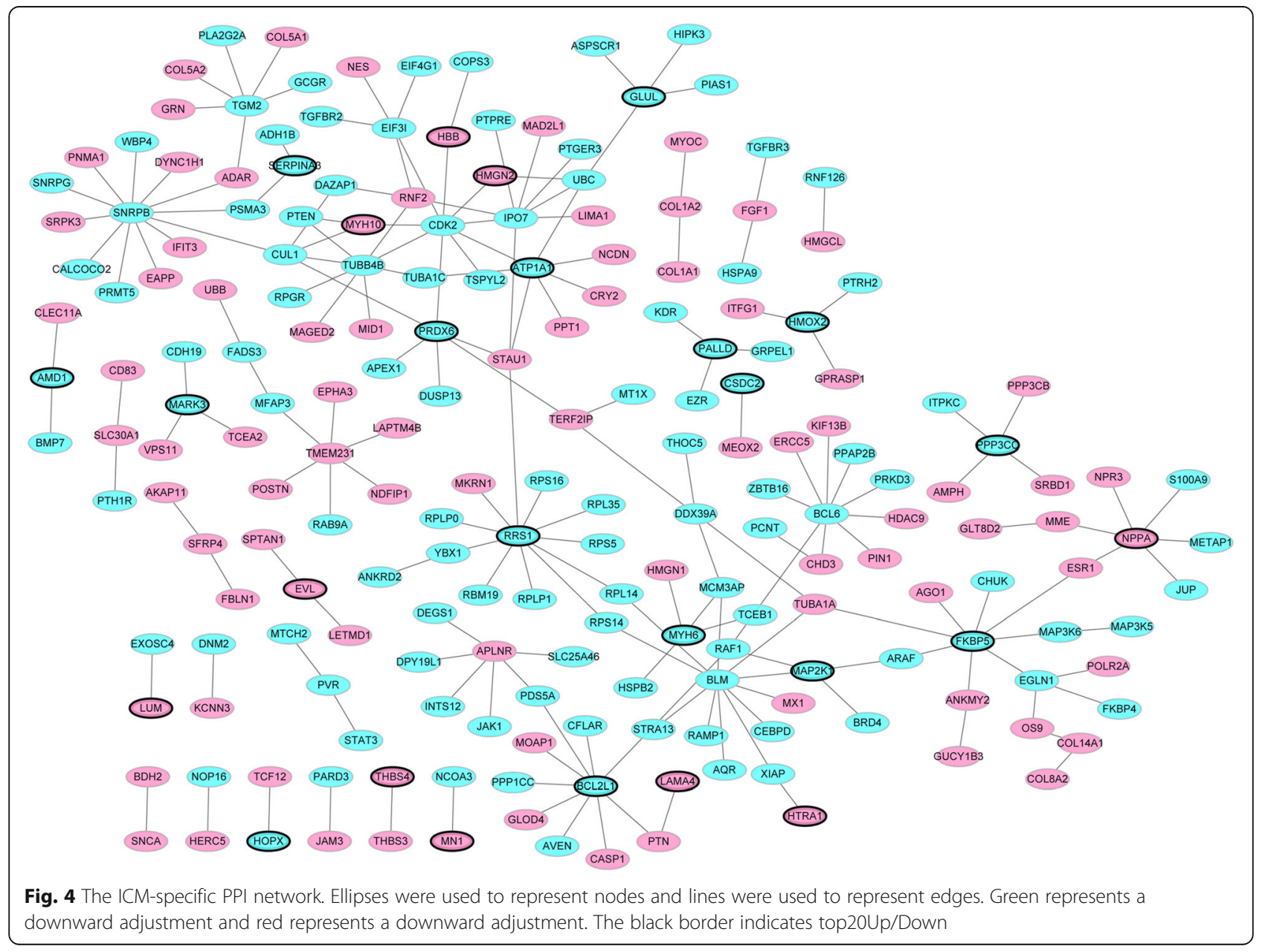




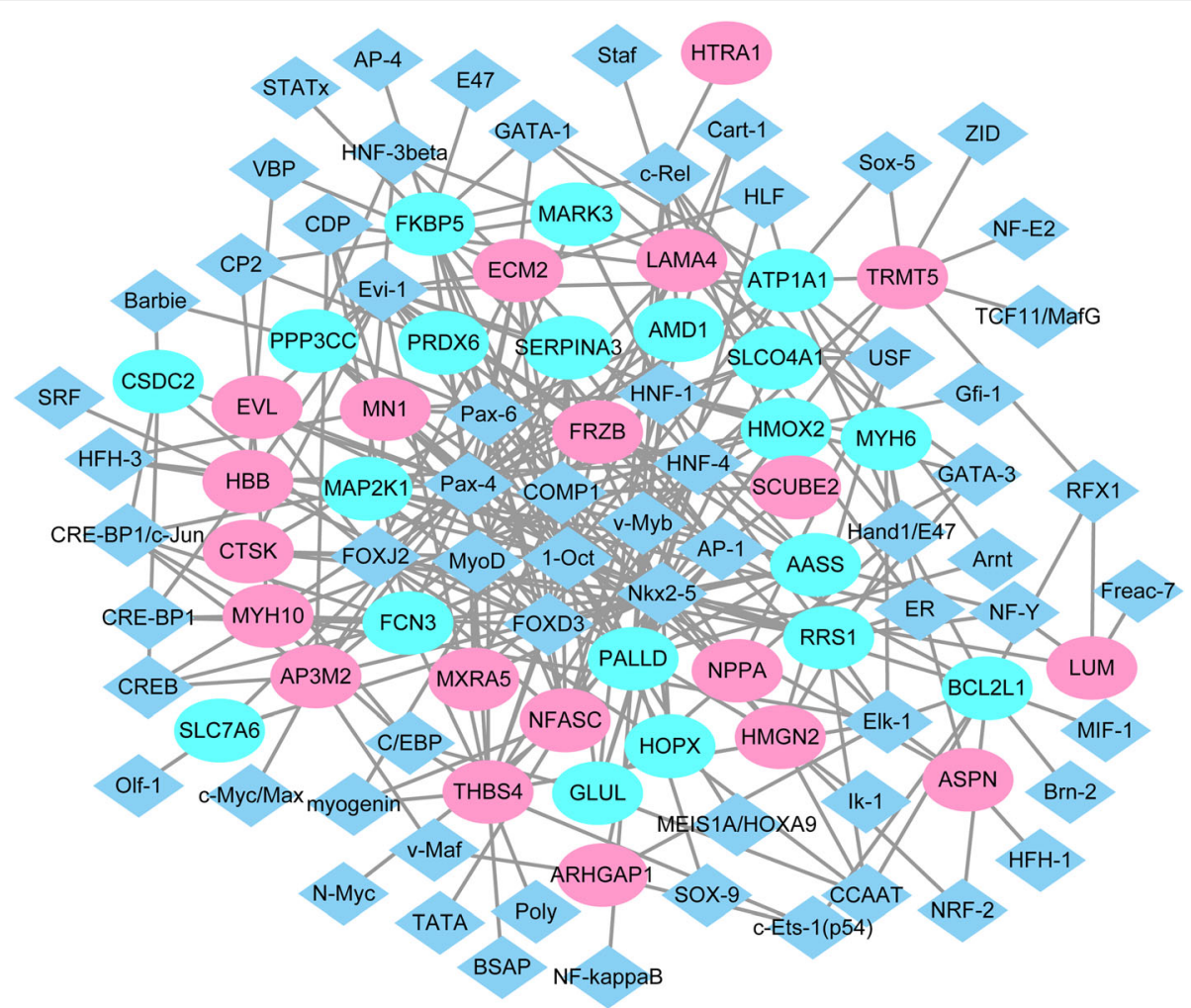

Fig. 5 ICM -specific transcriptional regulatory network. Ellipses and rhombus were used to represent nodes and lines were used to represent edges. The rhombus represented DEGs and the ellipses represented TFs. Green represents a downward adjustment and red represents a downward adjustment. The lines indicated TFs-DEGs pairs

BCL6, BLM, IPO7 and SERPINA3 were down-regulated and THBS4 was up-regulated in ICM compared with control. In generally, the validation results of qRT-PCR were consistent with our integration results and GSE116250 validation results.

\section{Discussion}

ICM continues to be one of the major diseases that threaten human health [1]. To make an accurate mechanism and find more effective therapeutic strategy for ICM in the early stage, it is need to find new therapeutic targets for ICM. With the emergence of highthroughput microarrays, a number of public resources have been build, among which the national center for biotechnology information (NCBI) GEO is the largest public resource [27]. Bioinformatics analysis based on GEO database provides valuable basis for revealing the pathogenesis of multiple diseases [28-30]. Integrated microarrays analysis with different platforms will obtain genome-wide expression profiling with larger sample size which will increase the statistical power than an individual microarray. Investigating abnormal gene expression in upstream TFs -mediated disease states can help to reveal the pathophysiological changes of complex diseases [31]. In the study, we carried out the integrated analysis of three gene expression datasets to identify the DEGs associated with ICM. A 1081 DEGs were identified in ICM with FDR $<0.05$. The ICM related pathways and TFs were also obtained by the bioinformatics methods. We selected MYH6, THBS4, BCL6, BLM, IPO7 and SERPINA3 to verify their expression in ICM. Expression of 6 DEGs (MYH6, THBS4, BCL6, BLM, IPO7 and SERPINA3) in qRT-PCR results were consistent with our GEO analysis, which adds evidence to the reliability of our results.

MYH6 encodes the alpha heavy chain subunit of cardiac myosin in the developing atria. It has been reported that mutations of MYH6 associated with hypertrophic and dilated cardiomyopathy [32, 33]. MYH6 was associated with congenital heart disease, and indicate that by increase mutation of MYH6 could be associated with congenital heart disease [34]. Mutations in the head domain of MYH6 play a pivotal role in the progress of familial secundum-type atrial septal defects [35]. Jiang et al. found that silencing of mutant MYH6 transcripts in mice inhibited hypertrophic cardiomyopathy [36]. Castellana et al. reported the desmoglein-2/desmocollin2/MYH6 mutations might determine a mild hypertrophic phenotype associated both to ventricular tachyarrhythmias and atrio-ventricular block [37]. Granados- 

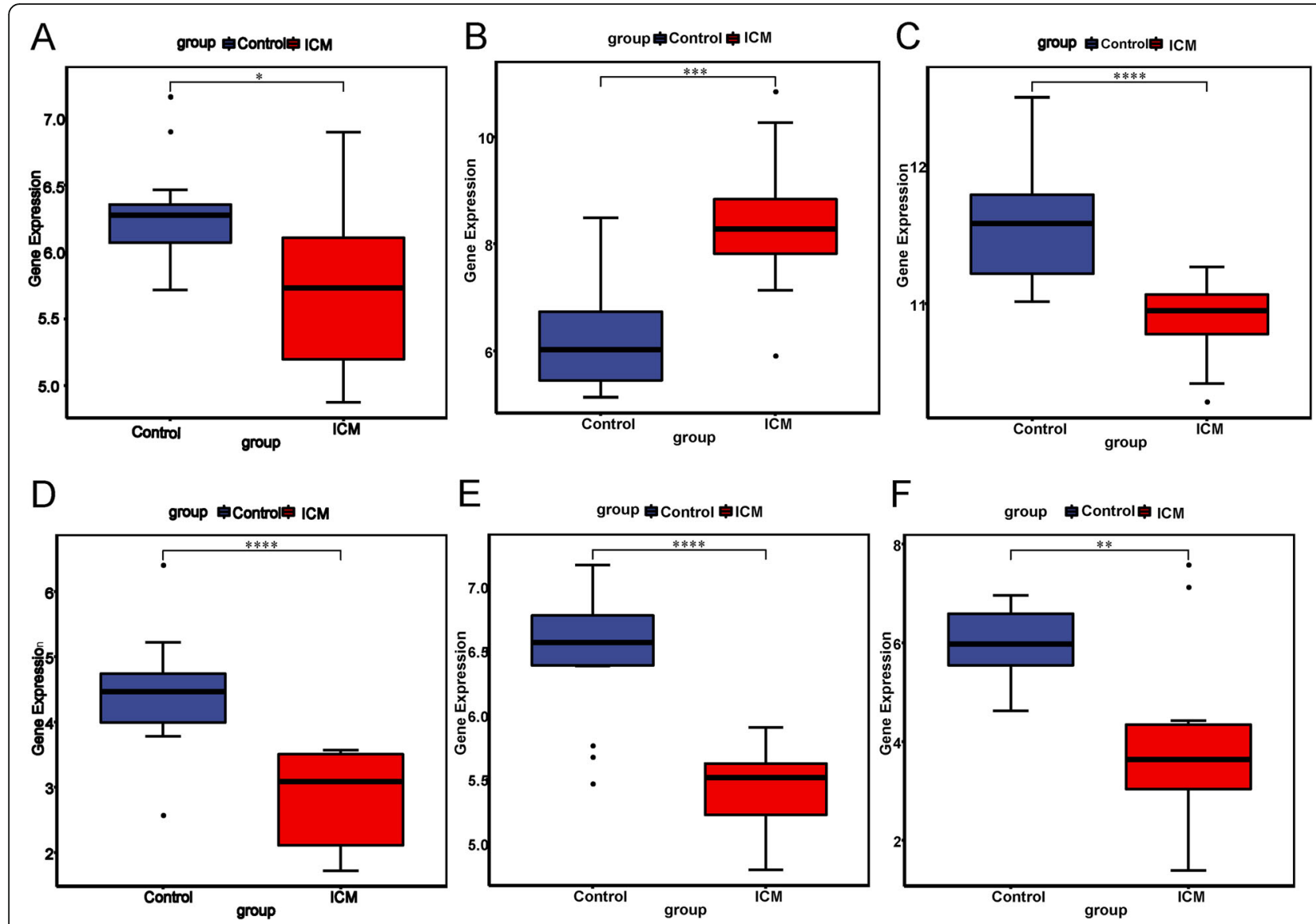

Fig. 6 Validation of selected DEGs in GSE116250. a MYH6. b THBS4. c BCL6. d BLM. e IPO7. f SERPINA3. The x-axis shows healthy normal control (blue colour) and ICM (red colour) groups and $y$-axis shows a log2 transformation to the intensities. ${ }^{*} P<0.05$, ${ }^{* *} P<0.01$, ${ }^{* *} P<0.001,{ }^{* * *} P<0.0001$

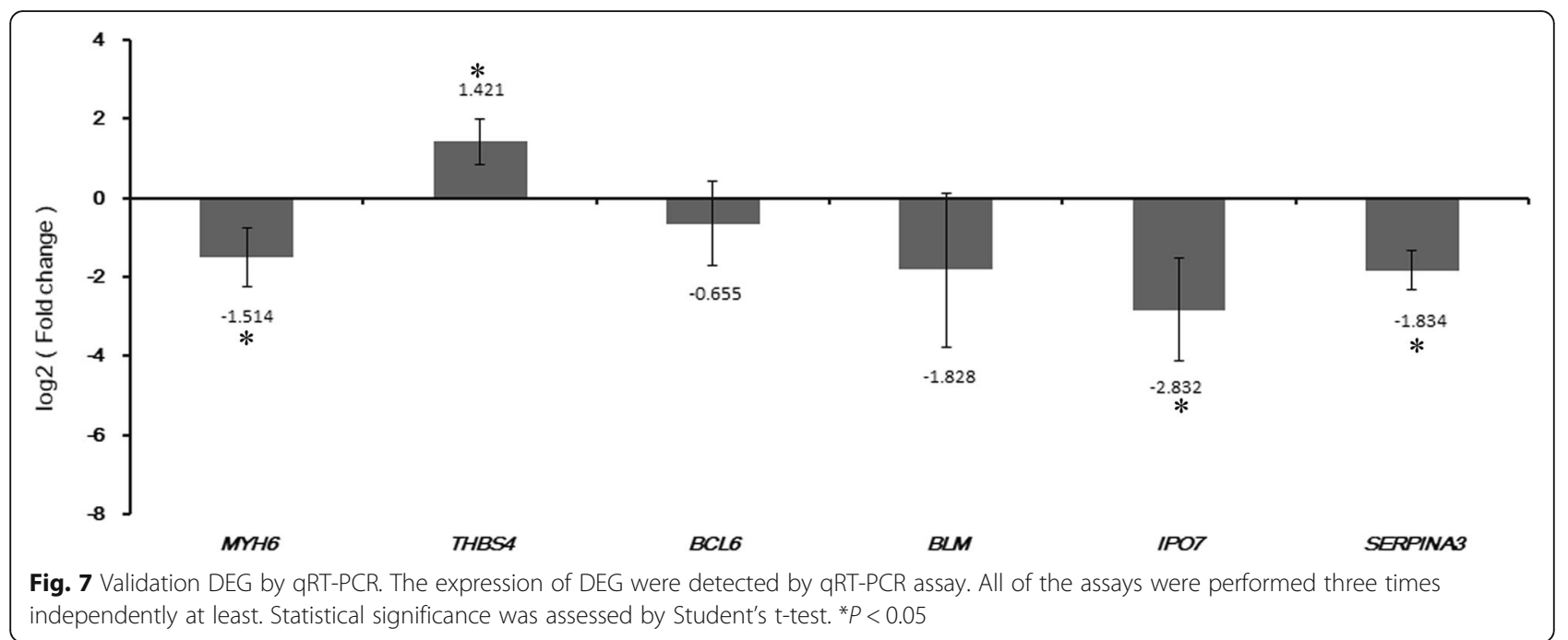


Riveron et al. reported that mutations of MYH6 affecting myofibril formation are associated with congenital heart defects, whereas others have identified mutations of the same gene in patients with hypertrophic and dilated cardiomyopathy [38]. Here, MYH6 was down-regulated in patient with ICM in both integration analysis and qRTPCR confirmation. The KEGG pathway enrichment analyses results showed that MYH6 was significantly enriched pathway of cardiac muscle contraction, hypertrophic cardiomyopathy and dilated cardiomyopathy. Therefore, we hypothesized that MYH6 might play key roles in ICM via regulating signaling pathway of cardiac muscle contraction, hypertrophic cardiomyopathy and dilated cardiomyopathy.

THBS4 is one of the exocrine glycoproteins involved in wound healing and tissue remodeling via modulating the repair and remodeling of the extracellular matrix [39, 40]. It has been found that THBS4 is continually abnormally expressed in the multiple solid cancers [41-43]. Recent research has indicated that THBS4 is involved in severe hypertrophic cardiomyopathy and heart failure pathogenesis [44]. In this study, THBS4 was one of top 6 TFs covering the most downstream DEGs, and was up-regulated in both integration analysis and qRT-PCR confirmation. The results displayed that THBS4 may play a key role in the pathogenesis of ICM. SERPINA3, a protease inhibitor, belongs to the superfamily of serine protease inhibitors. SERPINA3 is an acute phase response gene that is upregulated during inflammation [45]. Masanori et al. found that SERPINA3 may be novel diagnostic and pharmacological targets for heart failure [46]. SERPINA3 has been reported to be involved in the pathogenesis of myocardial ischemia-reperfusion injury [47]. Herein, SERPINA3 was one of top 40 DEGs, and was down-regulated in both integration analysis and qRT-PCR confirmation. Therefore, we hypothesized that SERPINA3 may be involved in the development of ICM.

However, this study has several limitations that need to be acknowledged. The small samples size (10 sample per group) for qRT-PCR confirmation might affect the quality of our results. Although the validation based on GSE116250 suggested that our qRT-PCR results were generally convincing, studies with larger sample size need to be conducted to confirm this conclusion. The identification of DEGs of ICM is a pilot study and further model systems or cell lines experiments are needed to reveal their biological functions in ICM.

\section{Conclusions}

The functional annotation, PPI network and ICMspecific transcriptional regulatory network were performed to identify DEGs, TFs and pathways in ICM which provides perspective to reveal the pathology and develop therapeutic targets for the ICM.

\section{Supplementary information}

Supplementary information accompanies this paper at https://doi.org/10. 1186/s12872-020-01596-w.

Additional file 1. All differentially expressed genes between ICM and healthy control.

\section{Abbreviations}

ICM: Ischemic cardiomyopathy; DEGs: Differentially expressed genes; PPI: Protein-protein interaction; qRT-PCR: Quantitative real time polymerase chain reaction; GEO: Gene expression omnibus; TFs: Transcription factors; FDR: False discovery rate; GO: Gene ontology; KEGG: Kyoto encyclopedia of genes and genomes; BioGRID: Biological general repository for interaction datasets; NCBI: National center for biotechnology information; HDL-C: Highdensity lipoprotein cholesterol; LDL-C: Low-density lipoprotein cholesterol; TG: Triglyceride; TC: Total cholesterol; HCT: Haematocrit; HB: Hemoglobin

\section{Acknowledgements}

None.

Authors' contributions

$H D, Y Y, X Z$ and $Y Z$ contributed to the conception of the study. HD and $Y Y$ contributed the materials and performed the experiment. $X Z$ and $Y Z$ performed the data analyses. HD, YY, XZ and $Y Z$ contributed significantly in writing the manuscript. All authors read and approved the final manuscript.

Funding

None.

Availability of data and materials

The datasets used and analysed during the current study are available from public database Gene Expression Omnibus repository. Accession numbers of the datasets used in current study are GSE46224, GSE52601, GSE5406 and GSE116250 in Gene Expression Omnibus (https://www.ncbi.nlm.nih.gov/geo).

\section{Ethics approval and consent to participate}

This study was approved by the ethical committee of the Capital medical university, Beijing Anzhen hospital. Written informed consent was obtained from all participants.

Consent for publication

The subjects gave written informed consent for the publication of any associated data.

\section{Competing interests}

The authors declare that they have no conflict of interest.

\section{Author details}

${ }^{1}$ Department of cardiac surgery, Capital medical university, Beijing Anzhen hospital, Beijing, China. ${ }^{2}$ Department of cardiology, Capital medical university, Beijing Anzhen hospital, No.2, Anzhen Road, Chaoyan District, Beijing 100029, China.

Received: 26 December 2019 Accepted: 24 June 2020

Published online: 06 July 2020

References

1. Schuster A, Morton G, Chiribiri A, Perera D, Vanoverschelde JL, Nagel E. Imaging in the management of ischemic cardiomyopathy: special focus on magnetic resonance. J Am Coll Cardiol. 2012;59(4):359-70.

2. Perera D, Clayton T, Petrie MC, Greenwood JP, O'Kane PD, Evans R, Sculpher M, McDonagh T, Gershlick A, de Belder M, et al. Percutaneous revascularization for ischemic ventricular dysfunction: rationale and design of the REVIVED-BCIS2 trial: percutaneous coronary intervention for ischemic cardiomyopathy. JACC Heart Fail. 2018;6(6):517-26.

3. Hasenfuss G, Pieske B. Calcium cycling in congestive heart failure. J Mol Cell Cardiol. 2002;34(8):951-69.

4. Cicconi S, Ventura N, Pastore D, Bonini P, Di NP, Lauro R, Marlier LN. Characterization of apoptosis signal transduction pathways in $\mathrm{HL}-5$ 
cardiomyocytes exposed to ischemia/reperfusion oxidative stress model. J Cell Physiol. 2003;195(1):27-37.

5. Yang H, Li H. CD36 identified by weighted gene co-expression network analysis as a hub candidate gene in lupus nephritis. PeerJ. 2019;7:e7722.

6. Wang Y, Wu N, Liu J, Wu Z, Dong D. FusionCancer: a database of cancer fusion genes derived from RNA-seq data. Diagn Pathol. 2015;10:131.

7. Sun Y, Lin J, Zhang L. The application of weighted gene co-expression network analysis in identifying key modules and hub genes associated with disease status in Alzheimer's disease. Ann Transl Med. 2019;7(24):800

8. Rajabi M, Kassiotis C, Razeghi P, Taegtmeyer H. Return to the fetal gene program protects the stressed heart: a strong hypothesis. Heart Fail Rev. 2007;12(3-4):331-43.

9. Li W, Li L, Zhang S, Zhang C, Huang H, Li Y, Hu E, Deng G, Guo S, Wang Y, et al. Identification of potential genes for human ischemic cardiomyopathy based on RNA-Seq data. Oncotarget. 2016;7(50):82063-73.

10. Roselló-Lletí E, Carnicer R, Tarazón E, Ortega A, Gil-Cayuela C, Lago F, González-Juanatey JR, Portolés M, Rivera M. Human ischemic cardiomyopathy shows cardiac Nos1 translocation and its increased levels are related to left ventricular performance. Sci Rep. 2016;6:24060.

11. Herrer I, Roselló-Lletí E, Ortega A, Tarazón E, Molina-Navarro MM, Triviño JC, Martínez-Dolz L, Almenar L, Lago F, Sánchez-Lázaro I. Gene expression network analysis reveals new transcriptional regulators as novel factors in human ischemic cardiomyopathy. BMC Med Genet. 2015;8(1):14.

12. Qiao A, Zhao Z, Zhang H, Sun Z, Cui X. Gene expression profiling reveals genes and transcription factors associated with dilated and ischemic cardiomyopathies. Pathol Res Pract. 2017;213(5):548-57.

13. Li GM, Zhang CL, Rui RP, Sun B, Guo W. Bioinformatics analysis of common differential genes of coronary artery disease and ischemic cardiomyopathy. Eur Rev Med Pharmacol Sci. 2018;22(11):3553-69.

14. Wang J, Wang F, Zhu J, Song M, An J, Li W. Transcriptome profiling reveals PHLDA1 as a novel molecular marker for ischemic cardiomyopathy. J Mol Neurosci. 2018;65(1):102-9.

15. Cortés R, Rivera M, Roselló-Lletí E, Martínez-Dolz L, Almenar L, Azorín I, Lago F, González-Juanatey JR, Portolés M. Differences in MEF2 and NFAT transcriptional pathways according to human heart failure Aetiology. PLoS One. 2012;7(2):e30915.

16. Pashmforoush M, Lu JT, Chen H, Amand TS, Kondo R, Pradervand S, Evans SM, Clark B, Feramisco JR, Giles W. Nkx2-5 pathways and congenital heart disease; loss of ventricular myocyte lineage specification leads to progressive cardiomyopathy and complete heart block. Cell. 2004;117(3):373-86.

17. Li C, Browder W, Kao RL. Early activation of transcription factor NF-kappaB during ischemia in perfused rat heart. Am J Physiol. 1999;276(2):543-52.

18. Haghikia A, Stapel B, Hoch M, Hilfikerkleiner D. STAT3 and cardiac remodeling. Heart Fail Rev. 2011;16(1):35-47.

19. Renata W, Julius M, Allison F, Alexander A, Wagner EF, Thierry P, Grzegorz S, Romeo R. The AP-1 transcription factor c-Jun prevents stress-imposed maladaptive remodeling of the heart. PLoS One. 2013;8(9):e73294.

20. Kugler KG, Mueller LA, Graber A, Dehmer M. Integrative network biology: graph prototyping for co-expression cancer networks. PLoS One. 2011;6(7):e22843.

21. Emmertstreib F, Dehmer M. Networks for systems biology: conceptual connection of data and function. IET Syst Biol. 2011;5(3):185-207.

22. Yang KC, Yamada KA, Patel AY, Topkara VK, George I, Cheema FH, Ewald GA Mann DL, Nerbonne JM. Deep RNA sequencing reveals dynamic regulation of myocardial noncoding RNAs in failing human heart and remodeling with mechanical circulatory support. Circulation. 2014;129(9):1009-21.

23. Akat KM, Moore-McGriff D, Morozov P, Brown M, Gogakos T, Correa Da Rosa J, Mihailovic A, Sauer M, Ji R, Ramarathnam A, et al. Comparative RNA-sequencing analysis of myocardial and circulating small RNAs in human heart failure and their utility as biomarkers. Proc Natl Acad Sci U S A. 2014;111(30):11151-6.

24. Hannenhalli S, Putt ME, Gilmore JM, Wang J, Parmacek MS, Epstein JA, Morrisey EE, Margulies KB, Cappola TP. Transcriptional genomics associates FOX transcription factors with human heart failure. Circulation. 2006;114(12):1269-76.

25. Sweet ME, Cocciolo A, Slavov D, Jones KL, Sweet JR, Graw SL, Reece TB, Ambardekar AV, Bristow MR, Mestroni L, et al. Transcriptome analysis of human heart failure reveals dysregulated cell adhesion in dilated cardiomyopathy and activated immune pathways in ischemic heart failure. BMC Genomics. 2018;19(1):812

26. Felker GM, Shaw LK, O'Connor CM. A standardized definition of ischemic cardiomyopathy for use in clinical research. J Am Coll Cardiol. 2002;39(2): 210-8.

27. Liu X, Qu J, Xue W, He L, Wang J, Xi X, Yin Y, Qu Y. Bioinformatics-based identification of potential microRNA biomarkers in frequent and non- frequent exacerbators of COPD. Int J Chron Obstruct Pulmon Dis. 2018;13: 1217-28.

28. Manchia M, Piras IS, Huentelman MJ, Pinna F, Zai CC, Kennedy JL, Carpiniello B. Pattern of gene expression in different stages of schizophrenia: Down-regulation of NPTX2 gene revealed by a meta-analysis of microarray datasets. Eur Neuropsychopharmacol. 2017;27(10):1054-63.

29. Huang Y, Zhu J, Li W, Zhang Z, Xiong P, Wang H, Zhang J. Serum microRNA panel excavated by machine learning as a potential biomarker for the detection of gastric cancer. Oncol Rep. 2018;39(3):1338-46.

30. Zhang Q, Chen W, Chen S, Li S, Wei D, He W. Identification of key genes and upstream regulators in ischemic stroke. Brain Behav. 2019;9(7):e01319.

31. Zhao B, Wang M, Xu J, Li M, Yu Y. Identification of pathogenic genes and upstream regulators in age-related macular degeneration. BMC Ophthalmol. 2017;17(1):102.

32. Carniel E, Taylor MR, Sinagra G, Di Lenarda A, Ku L, Fain PR, Boucek MM, Cavanaugh J, Miocic S, Slavov D, et al. Alpha-myosin heavy chain: a sarcomeric gene associated with dilated and hypertrophic phenotypes of cardiomyopathy. Circulation. 2005;112(1):54-9.

33. Hershberger RE, Norton N, Morales A, Li D, Siegfried JD, Gonzalez-Quintana J. Coding sequence rare variants identified in MYBPC3, MYH6, TPM1, TNNC1, and TNNI3 from 312 patients with familial or idiopathic dilated cardiomyopathy. Circ Cardiovasc Genet. 2010;3(2):155-61.

34. Razmara E, Garshasbi M. Whole-exome sequencing identifies R1279X of MYH6 gene to be associated with congenital heart disease. BMC Cardiovasc Disord. 2018;18(1):137.

35. Posch MG, Waldmuller $\mathrm{S}$, Muller M, Scheffold T, Fournier D, AndradeNavarro MA, De Geeter B, Guillaumont S, Dauphin C, Yousseff D, et al. Cardiac alpha-myosin (MYH6) is the predominant sarcomeric disease gene for familial atrial septal defects. PLoS One. 2011;6(12):e28872.

36. Jiang J, Wakimoto H, Seidman JG, Seidman CE. Allele-specific silencing of mutant Myh6 transcripts in mice suppresses hypertrophic cardiomyopathy. Science (New York, NY). 2013;342(6154):111-4.

37. Castellana S, Mastroianno S, Palumbo P, Palumbo O, Biagini T, Leone MP, De Luca G, Potenza DR, Amico CM, Mazza T, et al. Sudden death in mild hypertrophic cardiomyopathy with compound DSG2/DSC2/MYH6 mutations: revisiting phenotype after genetic assessment in a master runner athlete. J Electrocardiol. 2019:53:95-9.

38. Granados-Riveron JT, Ghosh TK, Pope M, Bu'Lock F, Thornborough C, Eason J, Kirk EP, Fatkin D, Feneley MP, Harvey RP. Alpha-cardiac myosin heavy chain (MYH6) mutations affecting myofibril formation are associated with congenital heart defects. Hum Mol Genet. 2010;19(20):4007-16.

39. Muppala S, Xiao R, Krukovets I, Verbovetsky D, Yendamuri R, Habib N, Raman P, Plow E, Stenina-Adognravi O. Thrombospondin-4 mediates TGFbeta-induced angiogenesis. Oncogene. 2017;36(36):5189-98.

40. Stenina-Adognravi O, Plow EF. Thrombospondin-4 in tissue remodeling. Matrix Biol. 2019:75-76:300-13.

41. Liu J, Cheng G, Yang H, Deng X, Qin C, Hua L, Yin C. Reciprocal regulation of long noncoding RNAs THBS4003 and THBS4 control migration and invasion in prostate cancer cell lines. Mol Med Rep. 2016;14(2):1451-8.

42. Kuroda K, Yashiro M, Sera T, Yamamoto Y, Kushitani Y, Sugimoto A Kushiyama S, Nishimura S, Togano S, Okuno T, et al. The clinicopathological significance of Thrombospondin-4 expression in the tumor microenvironment of gastric cancer. PLoS One. 2019;14(11):e0224727.

43. Wu H, Zhang G, Li Z, Ma J, Han X, Xiang T, Jiang X. Thrombospondin-4 expression as a prognostic marker in hepatocellular carcinoma. Gene. 2019; 696:219-24

44. Tsoutsman T, Wang X, Garchow K, Riser B, Twigg S, Semsarian C. CCN2 plays a key role in extracellular matrix gene expression in severe hypertrophic cardiomyopathy and heart failure. J Mol Cell Cardiol. 2013:62:164-78.

45. Kloth JN, Gorter A, Fleuren GJ, Oosting J, Uljee S, ter Haar N, Dreef EJ, Kenter GG, Jordanova ES. Elevated expression of SerpinA1 and SerpinA3 in HLA-positive cervical carcinoma. J Pathol. 2008;215(3):222-30.

46. Asakura M, Kitakaze M. Global gene expression profiling in the failing myocardium. Circ J. 2009;73(9):1568-76.

47. Liu Z, Hou L, Liu Y, Gong J. LncRNA GAS5 exacerbates myocardial ischemiareperfusion injury through regulating serpina3 by targeting miR-137. Int J Cardiol. 2020;306:9.

\section{Publisher's Note}

Springer Nature remains neutral with regard to jurisdictional claims in published maps and institutional affiliations. 